\title{
Three Dimensional Digitally Designed Surgical Guides in Esthetic Crown Lengthening: A Clinical Case Report with 12 Months Follow Up
}

\section{Saad Obaid Alazmi}

Department of Periodontology \& Oral Medicine, College of Dentistry, Qassim University, Burayadh, Qassim, Saudi Arabia
Correspondence: Saad Obaid Alazmi Department of Periodontology \& Oral Medicine, College of Dentistry, Qassim University, Prince Naif Street \#14,

Burayadh, Qassim, 5I452, Saudi Arabia

Tel +966-6380-0050

Email S.alazmi@qu.edu.sa
Introduction: The digital workflow has improved the quality of dental care. The integration of computer-aided design/computer-aided-manufacturing (CAD/CAM), cone-beamcomputer-tomography (CBCT) and three-dimensional (3D) printing offers significant advantages for treatment planning and allows the dentist to visualize the treatment outcomes before they are initiated. Digital dental technology has become reliable and affordable with many applications for surgical and restorative solutions.

Case Description: The aim of the presented case is to demonstrate the advantages of 3D digitally-designed surgical guides in periodontal surgery. The patient needed esthetic crown lengthening to correct the altered passive eruption. Digital impressions, digital smile design (DSD) and CBCT were used for treatment planning and fabricating of 3D printed surgical guides using a 3D printer. These surgical guides were then used clinically. The clinical outcomes of 12 months follow up were precise and the surgeries were straight-forward, with relatively short procedure time. This is consistent with published literature comparing digitally generated guides to free-hand procedures.

Conclusion: The digitally generated 3D printed guides are reliable tools to perform surgeries precisely. Digital planning software is an efficient communication tool with referring dentists.

Keywords: esthetic crown lengthening, 3D surgical guide, CAD/CAM, CBCT, 3D printing

\section{Introduction}

One of the common esthetic concerns of patients is an excessive gingival display (EGD) with a short clinical crown. It can be caused by gingival enlargement or altered passive eruption (APE). Esthetic crown lengthening or gingivectomy are the most clinical treatment modalities used to increase crown length and achieve proper esthetics results. ${ }^{1}$ Nowadays, cone-beam computed tomography (CBCT) is considered as an accurate and reliable alternative diagnosed method for APE. ${ }^{2}$

It is largely dependent on the position of the alveolar bone crest in relation to the cementoenamel junction (CEJ) to determine whether to perform bone resection or gingivectomy alone. If the crest of the bone is at the cementoenamel junction or coronal to the CEJ, osseous resective surgery is indicated. ${ }^{3}$

The workflow of restorative and surgical dentistry has been improved by innovative computer-aided design/computer-aided manufacturing (CAD/CAM). Using digital technology offers significant advantages in the treatment planning and allows the dentist to visualize the treatment outcomes before they are initiated. Digital dental 


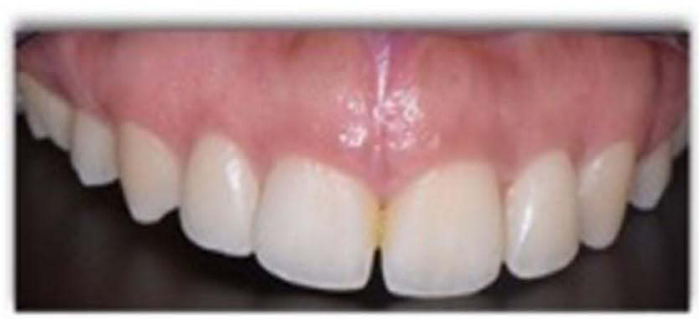

A

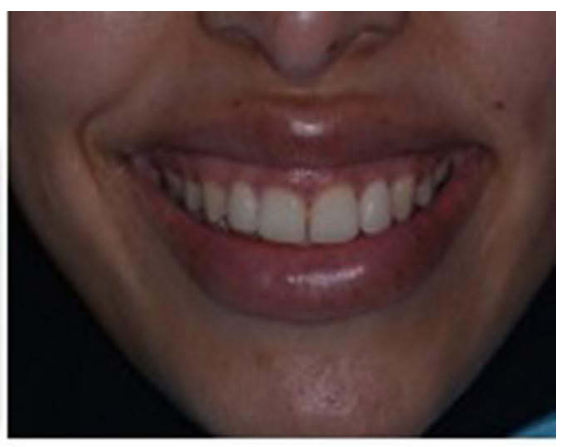

B

Figure I (A) Intraoral photography showing excessive gingival display, and (B) extraoral.

technology has become reliable and affordable with many applications for surgical and restorative workflows. ${ }^{4}$

With the integration of cone-beam computed tomography (CBCT), CAD/CAM technology and threedimensional (3D) printing, the periodontist could plan and perform esthetic crown lengthening surgery using a computer-generated guide, and trust the reliability of the guidance provided. The patient also benefits from having a shorter surgical time, with less morbidity, and a more precise outcome than via the conventional way. ${ }^{5}$

Digital smile design (DSD) is another software application that aids in diagnosis, planning and treatment at the esthetic zone and allows the patient to visualize the results of treatment before initiating it. By combining the use of DSD and $\mathrm{CAD} / \mathrm{CAM}$ technology with (3D) printing, a surgical guide for esthetic crown lengthening (ECL) could be produced with reliable accuracy. ${ }^{6}$ Therefore, the aim of the presented case is to demonstrate the advantages of $3 \mathrm{D}$ digitally-designed surgical guides in periodontal surgery and show the treatment stability and a follow up of 12 months.

\section{Case Report}

A 29-year-old female, medically fit, presented to University Dental Clinic complaining of dissatisfaction of her smile due to EGD. Pre-surgical evaluation included Intraoral (Figure 1A) and extraoral (Figure 1B) photography and video capture of dynamic smile positions. The patient provided informed consent to publish their case details and any accompanying images. Ethical approval to report the case was not needed per the Qassim University Institution Review Board.

\section{Design and 3D Printing of Surgical Guide}

An intraoral scanner camera $\left(\right.$ Omnicam $\left.{ }^{\circledR}\right)$ was used for the digital impression of the teeth and the occlusion. The case was diagnosed with EGD from tooth \#16-26, due to altered passive eruption and classified as Type1A, except \#14 and 13 are type1B, according to Coslet et al. ${ }^{7}$

The planning of the management was performed with the aid of a virtual planning technique in the DSD application (Figure 2), with the purpose of providing a better preoperative understanding of the proposed treatment, before commencement of active care. Then the esthetic crown lengthening guide was designed digitally (Figure 3). The 3D printer (ProJet MJP 3600 Dental 3D systems ${ }^{\circledR}$ ) was used to generate the surgical guide (Figure 4).

\section{Clinical Surgical Procedure}

Local anesthesia was applied by buccal infiltration technique. The 3D guide was utilized for the esthetic crown lengthening, with gingivoplasty incisions at the facial side of teeth \#16 to \#26 using internal bevel incision and blade 15C (Figure 5), then the collar of soft tissue was removed using a surgical curette (Figure 6). After sounding, flapless osteoectomy was selectively done at the facial bone of teeth \#13 and \#14 (Figure 7). PeriAcryl (cyanoacrylate adhesive dressing) was applied to control bleeding and enhance the healing (Figure 8).

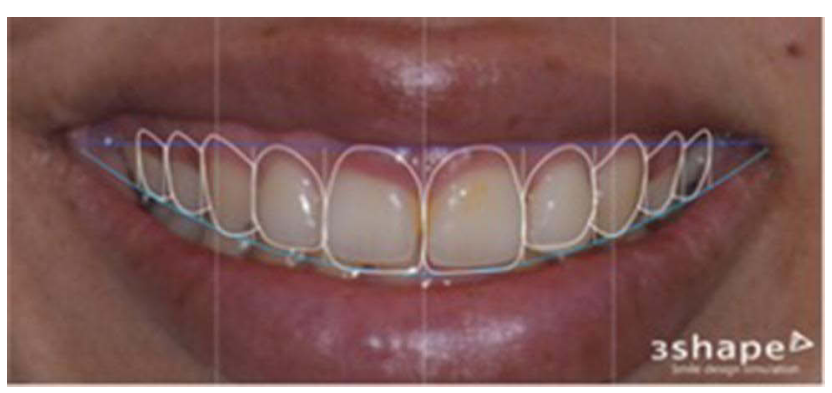

Figure 2 Virtual planning technique in the DSD application for planning of the management. 


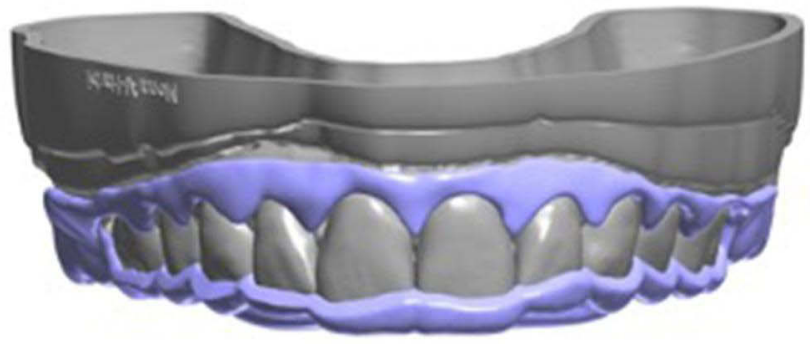

Figure 3 Designing the esthetic crown lengthening stent digitally.

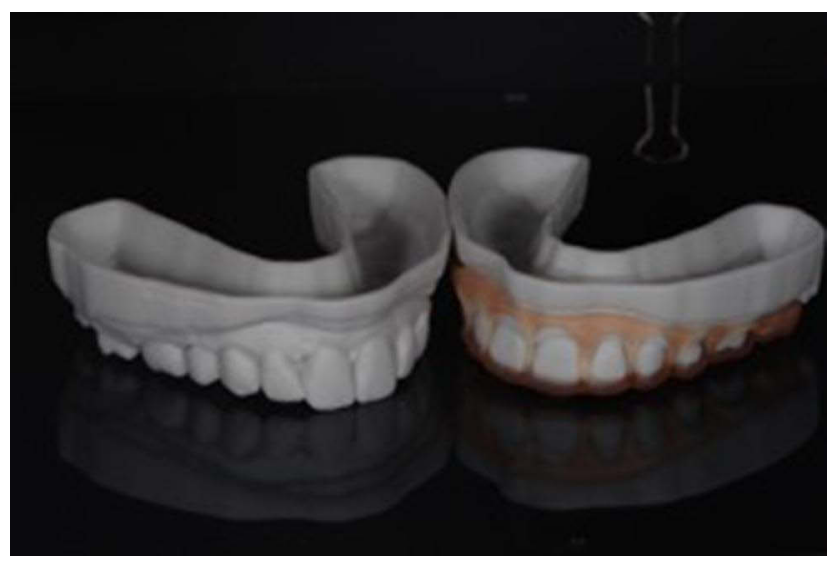

Figure 4 3D printed cast and surgical guide.

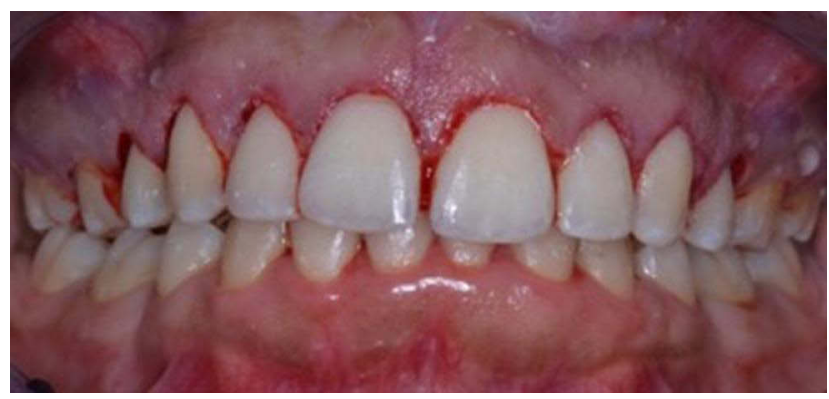

Figure 6 Postoperative clinical photo after gingivoplasty.

Postoperative management included oral hygiene instructions, pain medications and $0.2 \%$ chlorhexidine gluconate mouthwash twice daily. The evaluation of postoperative healing was done at intervals of 2, 4, and 6 weeks. The healing was uneventful (Figure 9.). The patient was pleased with the outcomes and placed on recall visits of 3, 6, and 12 months. After one year the patient came for a recall visit and the clinical photos were taken (Figure 10).

\section{Discussion}

Surgical guides, particularly when anterior teeth are involved, are typically developed as a guideline for crown lengthening

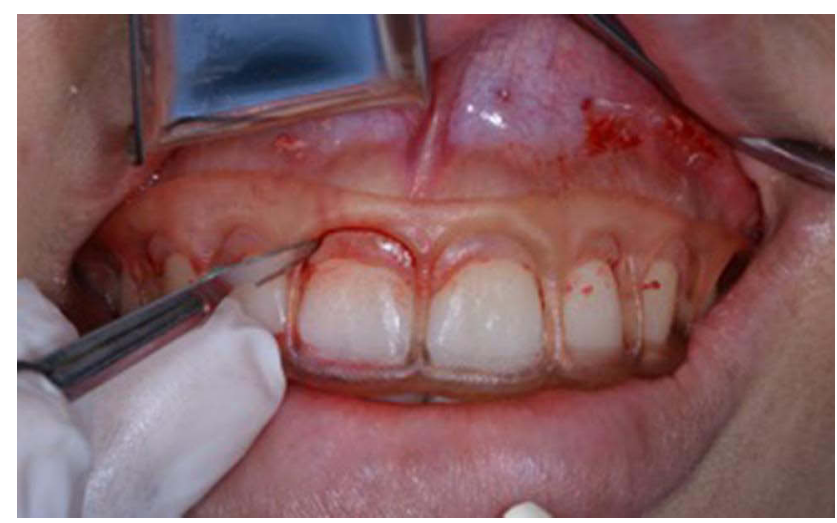

Figure 5 Gingivoplasty incision based on 3D guide.

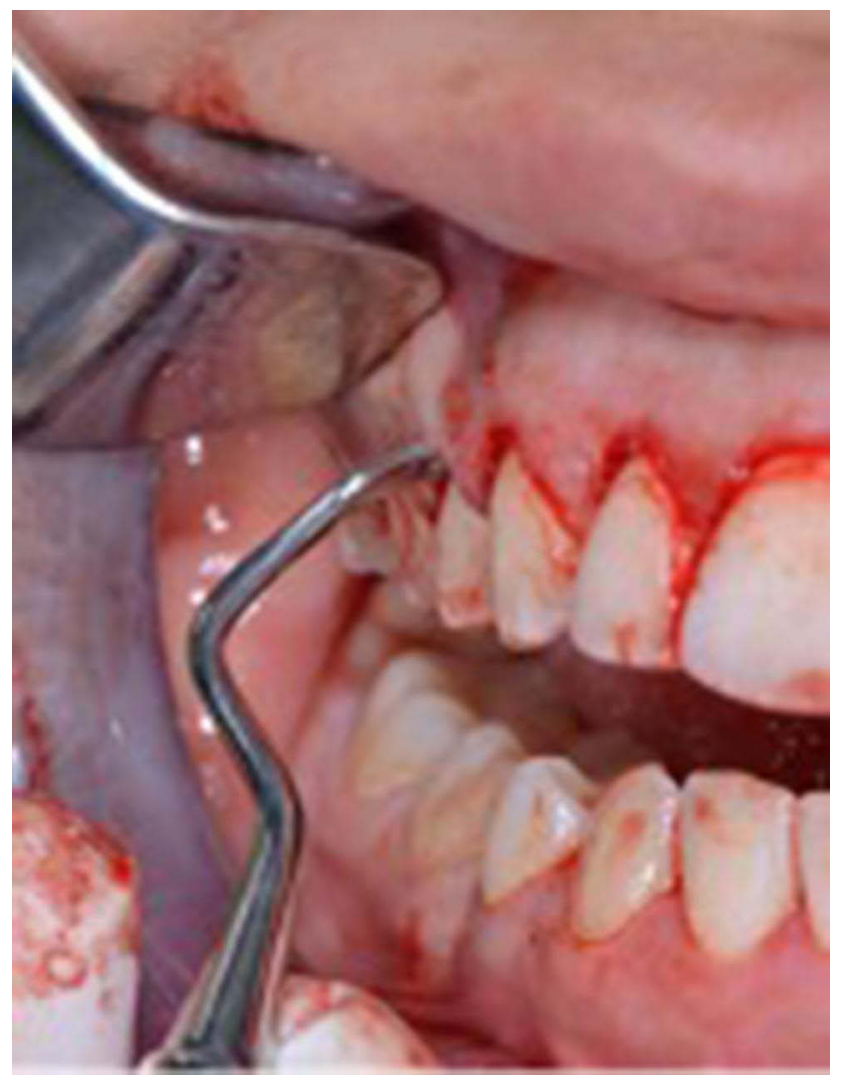

Figure 7 Flapless osteoectomy for tooth \#|4.

procedures. Diagnostic waxing is required to predict the potential length of the crown and to serve as a guide for soft tissue incision and freehand osteoectomy while using Vacuform shells or an acrylic mock-up. On the other hand, the using of digital technology in periodontal surgery offers instructions for both gingival and bone resection, thereby promoting the surgical process and producing predictable results. ${ }^{8}$

Passos et al use digital workflows to generate a surgical guide for the gingival margin position. ${ }^{9}$ They use a $3 \mathrm{D}$ 


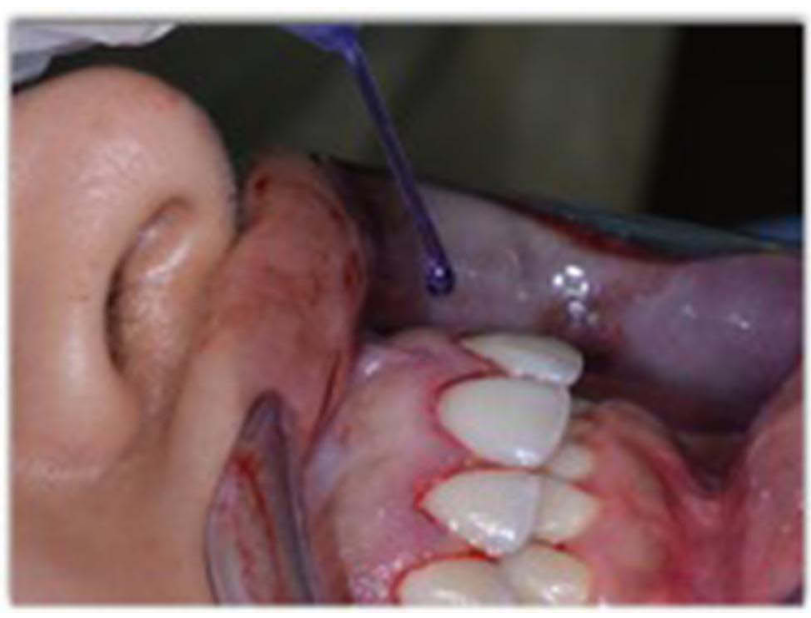

Figure 8 Postoperative, PeriAcryl applied.

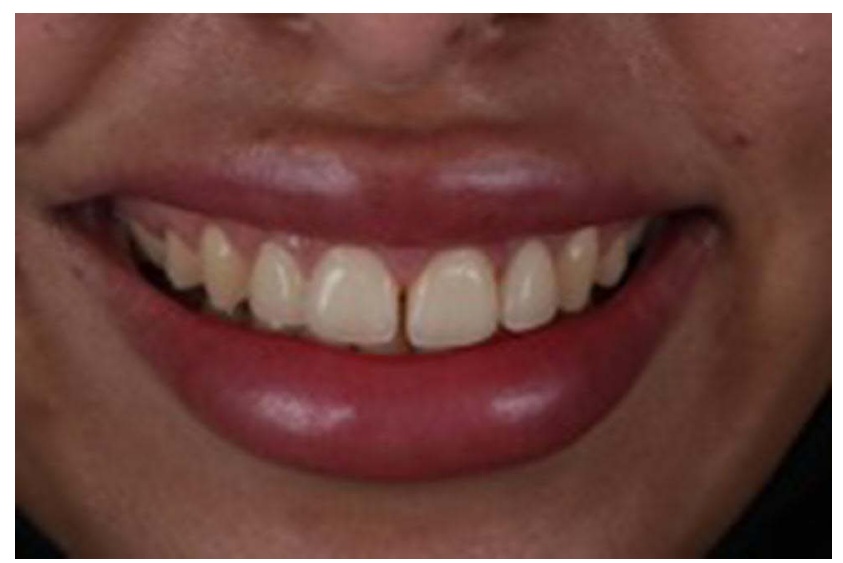

Figure 9 Showing postoperative healing after 6 weeks.

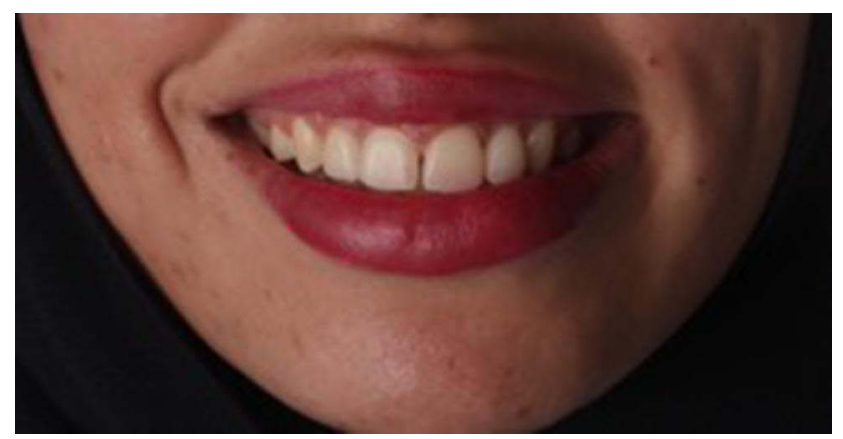

Figure 10 After 12 months follow up.

digital guide and find that it is reliable, accurate and reduces surgical time. This digital workflow controls the lab work instead of the old-style method of manual guide construction. This digital modern technique can easily manage the complicated periodontal case more accurately and quicker. Using a diagnostic wax-up procedure for esthetic crown lengthening can cause excessive removal of soft tissues and the chance of root exposure.

Using a three-dimensional digital guide in surgical implant placement, esthetic crown lengthening showed promising results in a recent review study. ${ }^{10}$ In our case report and follow-up for the 12 months come to conclude that the use of a digital workflow can decrease the chances of hard and soft tissue under- or overcontouring. The 12 months which followed showed complete healing with an optimum result and stability of treatment outcomes with high patient satisfaction. This digital workflow guide detects the location of the cementoenamel junction and guides the gingivectomy and osteoectomy, which decreases and controls the risk of hard and soft tissue under- or over-contouring. Limited literature is available, therefore we suggest the need for further clinical trials.

Despite the successful result of this technique it has a few limitations. Firstly, the material and equipment are expensive, which could not be easily provided by another clinic or afforded by the patient. Secondly, since it is a sensitive technique it needs skilled and well-trained lab technicians. Finally, treatment planning may take longer than planning for traditional surgery.

\section{Conclusion}

The digitally generated 3D printed guides can save time and aid the periodontist to plan and perform the ECL. Digital planning software is an efficient communication tool with referring dentists.

\section{Disclosure}

The author reports no conflicts of interest in this work.

\section{References}

1. Hempton TJ, Dominici JT. Contemporary crown-lengthening therapy: a review. J Am Dent Assoc. 2010;141(6):647-655. PMID: 20516094. doi:10.14219/jada.archive.2010.0252

2. Batista EL Jr, Moreira CC, Batista FC, de Oliveira RR, Pereira KK. Altered passive eruption diagnosis and treatment: a cone beam computed tomography-based reappraisal of the condition. $J$ Clin Periodontol. 2012;39(11):1089-1096. PMID: 22966787. doi:10.1111/ j.1600-051X.2012.01940.x

3. Mele M, Felice P, Sharma P, Mazzotti C, Bellone P, Zucchelli G. Esthetic treatment of altered passive eruption. Periodontol 2000. 2018;77(1):65-83. PMID: 29504162. doi:10.1111/prd.12206

4. Lin WS, Zandinejad A, Metz MJ, Harris BT, Morton D. Predictable restorative work flow for computer-aided design/computer-aided manufacture-fabricated ceramic veneers utilizing a virtual smile design principle. Oper Dent. 2015;40(4):357-363. PMID: 25706616. doi:10.2341/13-295-S 
5. Liu X, Yu J, Zhou J, Tan J. A digitally guided dual technique for both gingival and bone resection during crown lengthening surgery. $J$ Prosthet Dent. 2018;119(3):345-349. PMID: 28689907. doi:10.1016/j.prosdent.2017.04.018

6. Ruhmann NL, Borrasca C, Araújo CA, Pfau EA. Aesthetic rehabilitation of the "gummy smile" associated to virtual planning with "digital smile design"-DSD. J Surg Clin Dent. 2014;1(1):5-9.

7. Coslet JG, Vanarsdall R, Weisgold A. Diagnosis and classification of delayed passive eruption of the dentogingival junction in the adult. Alpha Omegan. 1977;70(3):24-28. PMID: 276255.

8. Alhumaidan A, Alqahtani A, al-Qarni F. 3D-printed surgical guide for crown lengthening based on cone beam computed tomography measurements: a clinical report with 6 months follow up. Appl Sci. 2020;10(16):5697. doi:10.3390/app10165697
9. Passos L, Soares FP, Choi IGG, Cortes ARG. Full digital workflow for crown lengthening by using a single surgical guide. $J$ Prosthet Dent. 2020;124(3):257-261. PMID: 31787273. doi:10.1016/j. prosdent.2019.06.027

10. Alazmi SO. Three dimensional digitally designed surgical guides in periodontal and implant surgeries: a review. Int J Dent Sci Res. 2020;8(6):150-154. doi:10.12691/ijdsr-8-6-2

Clinical, Cosmetic and Investigational Dentistry

\section{Publish your work in this journal}

Clinical, Cosmetic and Investigational Dentistry is an international, peer-reviewed, open access, online journal focusing on the latest clinical and experimental research in dentistry with specific emphasis on cosmetic interventions. Innovative developments in dental materials, techniques and devices that improve outcomes and patient satisfaction and preference will be highlighted. The manuscript management system is completely online and includes a very quick and fair peer-review system, which is all easy to use. Visit http://www.dovepress.com/testimonials.php to read real quotes from published authors. 